\title{
Long-Range Zero-Power Multi-Sensing in Industrial Environment using Polarization Diversity and 3D Radar Imagery
}

\author{
Dominique Henry $^{\# 1}$, Timothée Marchal ${ }^{\# 2}$, Julien Philippe ${ }^{\# 3}$, Hervé Aubert ${ }^{\# 4}$, Patrick Pons ${ }^{\# 5}$ \\ ${ }^{\#}$ LAAS-CNRS, Université de Toulouse, CNRS, INPT, Toulouse, France \\ 1 dhenry@laas.fr, ${ }^{2}$ tmarchal@ @laas.fr, ${ }^{3}$ jphilipp@laas.fr, ${ }^{4}$ ppons@ laas.fr, ${ }^{5}$ haubert@laas.fr,
}

\begin{abstract}
This paper describes the wireless and simultaneous interrogation of multiple passive (zero-power) pressure sensors in an industrial environment with a reading range of at least 3 meters. The 3D beamscanning of the scene is performed from a $24 \mathrm{GHz}$ FM-CW radar and for diverse electric field polarizations. The benefits of using the cross-polarized electric fields combined with a radar imagery technique are enlightened from the analysis of the ambient clutter and the simultaneous remote interrogation of two passive pressure sensors. The measurement uncertainty on pressure obtained from the proposed long-range wireless technique is finally reported and discussed.
\end{abstract}

Keywords - passive microwave remote sensing, radar imaging, radar remote sensing, transducers, radar polarimetry

\section{INTRODUCTION}

Remote sensing in electromagnetic reflective and/or harsh environments is challenging, especially when sensors are chipless and batteryless (or zero-power). Paradoxically, these sensors might be very useful in such environments, in which integrated circuits may be damaged and human intervention is limited. Remote sensing using Surface Acoustic Wave (SAW) tags, passive RFID tags or radiofrequency (RF) resonators has been investigated in harsh conditions, such as, in extreme temperatures [1][2] or cluttered environments [3]. The lifetime of tags in extreme conditions depends mainly on their constitutive materials, but the reliability of the wireless link between tags and their reader may be significantly degraded by surrounded electromagnetic reflective environments. The electromagnetic clutter (spurious radar echoes), multiple reflections (multipath) or destructive interferences (shadow regions) may decrease the signal-to-noise ratio and eventually make tags undetectable. One solution for increasing the signal-to-noise ratio is to take advantage of the polarization diversity. Benefits of using cross-polarized electric fields have been previously investigated for chipless RFID sensor tags in [4], [5], [6]. However, these previous studies targeted short reading ranges (few tens of centimeters). In many industrial applications, such as Structural Health Monitoring (SHM) in inaccessible areas, larger range of wireless interrogation is often required. Cross-polarized sensor tags combined with a microwave radar imagery technique allow reaching radar-to-tag distance of 50 meters in indoor environments [7]. In this paper, we propose a new long-range wireless multi-sensing technique in industrial environments using zero-power (passive or batteryless) pressure sensors and cross-polarized electric fields. The term long-range refers

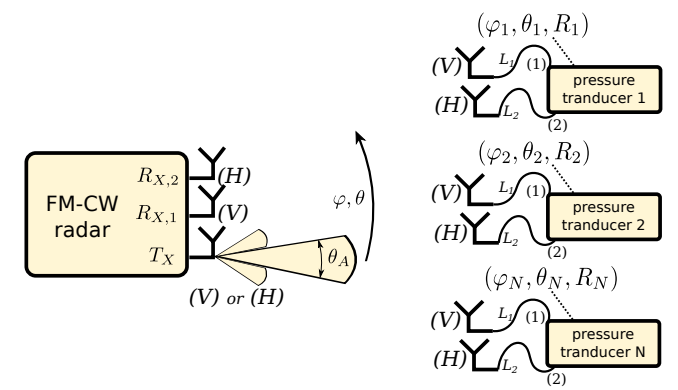

Fig. 1. Schematic of the 3D beamscanning principle of $N$ zero-power sensors with a FM-CW radar using polarization diversity. Pressure sensors are here passive pressure transducers connected to two cross-polarized antennas through delay lines.

here to a distance of interrogation of 3 meters or more, while the term short-range refers to reading ranges of few centimeters usually offered by standard chipless RFID sensor tags. Moreover, the term multi-sensing refers here to the simultaneous remote interrogation of multiple sensors in the scene. The so-called testing loop is used to measure and characterize the flow of fluids inside pipes, and many invasive sensors are usually needed to measure, e.g., the temperature and pressure of the flow inside pipes. These sensors are generally located along pipes typically of few tens meters long. In this paper, we investigate the radar interrogation of several passive pressure sensors surrounded by many electromagnetic reflective structures (such as metallic pipes, grids, walls, etc.).

The paper is organized as follows: Section II details the 3D beamscanning principle using co- and cross-polarized electric fields. In Section III, the experimental setup is described and two zero-power pressure sensors next to a testing loop are wirelessly interrogated. Next, clutter and radar echoes of these sensors are analyzed from 3D radar images and the computation of the so-called isolines. Finally, the remote estimation of the overpressure is reported and the measurement uncertainty obtained from the proposed wireless technique is finally given and discussed.

\section{MEASUREMENT PRINCIPLE}

The measurement principle is illustrated on Fig. 1. The reader unit is a FM-CW radar with carrier frequency $f_{c}$ and bandwidth $B$. The front-end is composed of the transmission channel $\left(T_{x}\right)$ and two reception channels $\left(R_{x, 1}\right.$ and $\left.R_{x, 2}\right)$. The $T_{x}$ antenna has narrow beamwidth in azimuth and elevation, denoted respectively by $\varphi_{A}$ and $\theta_{A}$, and its polarization is either vertical $(V)$ or horizontal $(H)$. The 
$R_{x, 1}$ antenna is vertically-polarized, while the polarization of the $R_{x, 2}$ antenna is horizontal. The radar performs the beamscanning of the scene, in which are distributed $N$ zero power pressure (or batteryless) sensors at respective positions $\left(\varphi_{1}, \theta_{1}, R_{1}\right),\left(\varphi_{2}, \theta_{2}, R_{2}\right), \ldots,\left(\varphi_{N}, \theta_{N}, R_{N}\right)$ relatively to the FM-CW radar location. The triplet $\left(\varphi_{k}, \theta_{k}, R_{k}\right)$ designates the azimuth, elevation and range from the radar of the $k^{t h}$ pressure sensor. As illustrated in Fig. 1, each sensor is composed of : (i) A zero-power pressure transducer, that is, a passive two-port which converts the pressure applied on the sensor into the variation of its scattering parameters $S_{11}, S_{12}, S_{21}, S_{22}$ at the radar operating frequency; (ii) $V-$ and $H$-polarized antennas; (iii) Two delay lines of electrical lengths $L_{1}$ and $L_{2}$, which are used for connecting the $V-$ and $H$-polarized antennas to the input (1) and output (2) ports of the transducer. The analysis of the so-called backscattering antenna (or sensing) mode of such passive and wireless sensors allows in principle the remote estimation of the overpressure. This electromagnetic backscattering originates in the impedance mismatch at input and output ports of the transducer : as the mismatch level depends on the pressure applied on the transducer, overpressure variation is expected to change the radar echo level of the sensor. The electric field transmitted by the radar is here either $H-$ or $V$-polarized, while the sensing mode is dual-polarized, that is, the electric field backscattered by the sensor is the combination of $H$-polarized and $V$-polarized electric fields. When the electric field transmitted by the radar is, say, $H$-polarized, the $H$-polarized component of the backscattered electric field is due to the sensing and structural modes of the $H$-polarized antenna of the sensor. Moreover, the $V$-polarized component of the field backscattered by the sensor is generated by the radiation from the $V$-polarized sensor antenna of the electric field received by the $H$-polarized sensor antenna and transmitted through the transducer and delay lines. The resulting $V$-polarized component of the backscattered electric field is next received by the $R_{x, 1}$-antenna.

Table 1. Expected range from the radar of the backscattering sensing mode of the $k^{t h}$ pressure sensor as a function of the polarization configuration $p$.

\begin{tabular}{|l|c|c|c|}
\hline \multicolumn{2}{|c|}{} & \multicolumn{2}{c|}{ Polarization of the radar $T_{x}$ antenna } \\
\cline { 2 - 4 } \multicolumn{2}{|l|}{} & $V$-pol & $H$-pol \\
\hline $\begin{array}{l}\text { Polarization } \\
\text { of the } \\
\text { radar } R_{x} \\
\text { antennas }\end{array}$ & \multirow{2}{*}{ a-pol } & $p=V V$ & $p=V H$ \\
& & $R_{k}+L_{1}$ & $R_{k}+\frac{L_{1}+L_{2}}{2}$ \\
\cline { 2 - 4 } & $H$-pol & $p=H V$ & $p=H H$ \\
$R_{k}+\frac{L_{2}+L_{1}}{2}$ & $R_{k}+L_{2}$ \\
\hline
\end{tabular}

In order to clarify the various polarization configurations studied in this paper, the so-called polarization configuration, denoted by $p$, is defined here as follows: when the electric field transmitted by the radar is $H$-polarized (resp. $V$-polarized), then (i) $p=H V$ (resp. $p=V V$ ) if the electric field is received by the $R_{x, 1}$-antenna, and (ii) $p=H H$ (resp. $p=V H)$ if the electric field is received by the $R_{x, 2}$-antenna. Throughout this paper, $p=V V$ or $H H$ refers to the co-polarization (co-pol) configuration, while $p=V H$ or

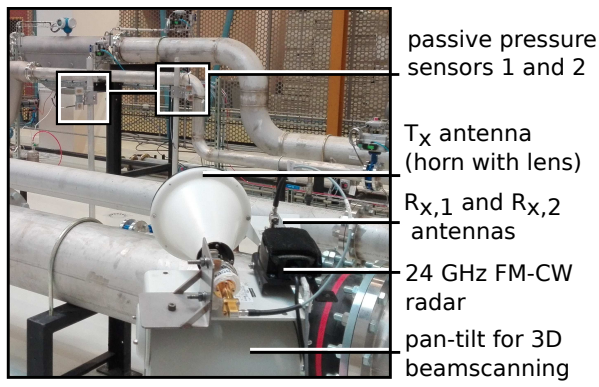

Fig. 2. Two passive pressure sensors located respectively at $R_{1}=3.3 \mathrm{~m}$ and $R_{2}=4.9 \mathrm{~m}$ from the $24 \mathrm{GHz}$ FM-CW radar in an industrial hangar. Sensors are surrounded by many electromagnetic reflective structures (metallic pipes, grid, walls, etc.)

$H V$ refers to the cross-polarization (x-pol) configuration. The eventual electromagnetic coupling between the two sensors antennas is assumed to be negligible. As reported in Table 1 , it is expected that the distance between the radar and the backscattering sensing mode will depend on the chosen polarization configuration.

\section{MATERIAL AND Method}

To demonstrate the feasibility of the multisensing in cluttered environments based on co- and cross-polarized electric fields and 3D radar imagery, two pressure sensors, named sensor 1 and sensor 2, are placed next to a testing loop in an industrial hangar (operating mode of such sensors can be found in [8]). An overpressure is first applied on the two sensors from 0 bar to 2 bars. As depicted in Fig. 2 , these sensors are located respectively at ranges $R_{1}=3.3 \mathrm{~m}$ and $R_{2}=4.9 \mathrm{~m}$ from the FM-CW radar of carrier frequency $f_{c}=23.8 \mathrm{GHz}$ and modulation bandwidth $B=2 \mathrm{GHz}$. The radar $T_{x}$-antenna is a circular horn with a dielectric lens, and its gain is of $28 \mathrm{dBi}$ with same beamwidth in azimuth and elevation $\left(\varphi_{A}=\theta_{A}=6^{\circ}\right) . R_{x, 1}$ and $R_{x, 2}$ antennas are rectangular horns with a gain of $20 \mathrm{dBi}$. The output power of the radar front-end is of $10 \mathrm{dBm}(10 \mathrm{~mW})$. The two antennas connected to pressure sensors are identical cross-polarized rectangular horns (gain of 20dBi). As electrical lengths of the delay lines are $L_{1}=L_{2}=1.2 \mathrm{~m}$, it can be derived from Table 1 that sensing modes are located at the same distance from the radar for all polarization configurations. Sensing modes of the sensor 1 and sensor 2 are located respectively at $4.5 \mathrm{~m}$ and $6.1 \mathrm{~m}$ from the radar. The radar is mounted on a pan-tilt plateform that performs a 3D mechanical beamscanning of the scene. The angular resolution is $1^{\circ}$ both in azimuth and elevation. The range resolution $d$ is of $\frac{c}{2 B}$, where $c$ is the speed of light. As a result, $d=7,5 \mathrm{~cm}$. To obtain $3 \mathrm{D}$ radar images for any polarization configuration $p(=V V, V H, H V$ and $H H)$, the beamscanning is performed using a $V$-polarized transmitted electric field, and next by using a $H$-polarized transmitted field. Scattering parameters of the two pressure transducers are displayed in Fig. 3a for the sensor 1 and in Fig. 3b for the sensor 2 .

Isolines of the beamscanned scene are computed for 3D radar images in elevation and azimuth planes. The term isoline refers to the line along which the radar echo level 


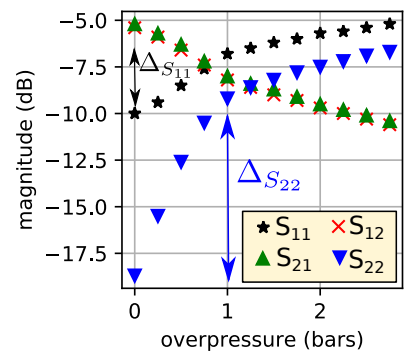

(a)

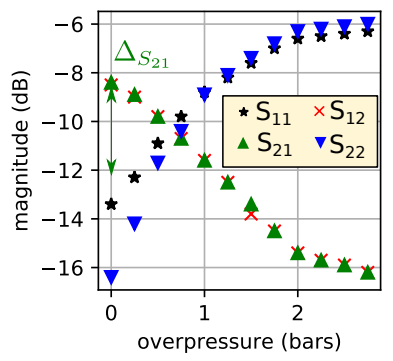

(b)
Fig. 3. Measured scattering parameters at $23.8 \mathrm{GHz}$ of (a) sensor 1 and (b) sensor 2 as a function of the applied overpressure. $\Delta_{S_{q}}$ (where $q=11,12,21$ and 22) denotes the full-scale measurement range of the scattering parameter $S_{q}$

is the same.Computed isolines associated with the clutter are displayed in Fig. 4 in black color for $p=V V, V H, H V$, and $H H$ in $(\theta, \varphi, R)$ coordinate system. It can be observed that the number of isolines differs significantly between co-pol and x-pol configurations: the number of isolines is actually of 391 for $p=V V, 66$ for $p=V H, 72$ for $p=H V$, and 353 for $p=H H$. This is due to the reduction of the clutter in the $\mathrm{x}$-pol radar images compared with one obtained from co-pol radar images. In red and blue colors are displayed in Fig. 4 isolines of sensing modes for the two passive pressure sensors. During the beamscanning, the applied overpressures were of 0,7 bar for the sensor 1 , and 1,1 bars for the sensor 2. The computed isolines allow estimating the location of these sensors with angular resolutions of $1^{\circ}$ in azimuth and elevation, and range resolution of $d=7.5 \mathrm{~cm}$. Inside the volume of the $3 \mathrm{D}$ scene beamscanned by the radar in a given polarization configuration, the sensors-to-clutter radar echoes ratio $\Lambda_{p}$ is computed as follows :

$$
\Lambda_{p}=\frac{N_{\text {sensors }, p}}{N_{\text {sensors }, p}+N_{\text {clutter }, p}}
$$

where $p$ denotes the polarization configuration, $N_{\text {sensor } s, p}$ is the number of isolines generated by the sensors and $N_{\text {clutter, } p}$ is the number of isolines generated by the clutter. In absence of clutter in the beamscanned volume, $\Lambda_{p}$ is equal to 1 and all isolines in this volume are generated only by the sensing mode. Consequently, the applied overpressure can be derived from the analysis of the isolines, as it will be shown below. However, when the clutter is very high, $\Lambda_{p}$ is close to 0 and the isolines are generated by the clutter only. As a consequence, the sensing modes are no more detectable and the overpressure cannot be remotely estimated. From the computed isolines displayed in Fig. 4, the following sensors-to-clutter radar echoes ratios are derived: $\Lambda_{V V}=0,0051, \Lambda_{V H}=0,030, \Lambda_{V H}=0,027$ and $\Lambda_{H H}=0,0056$. The x-pol ratios $\Lambda_{V H}$ and $\Lambda_{H V}$ are then ten times larger than the co-pol ratios $\Lambda_{V V}$ and $\Lambda_{H H}$. Therefore, compared with the co-pol configurations, the x-pol configurations allow detecting more easily the backscattering sensing modes in presence of strong electromagnetic clutter.

We show now that the overpressure applied on multiple sensors can be derived from the isolines generated by sensing modes. For this purpose, we define the statistical estimator
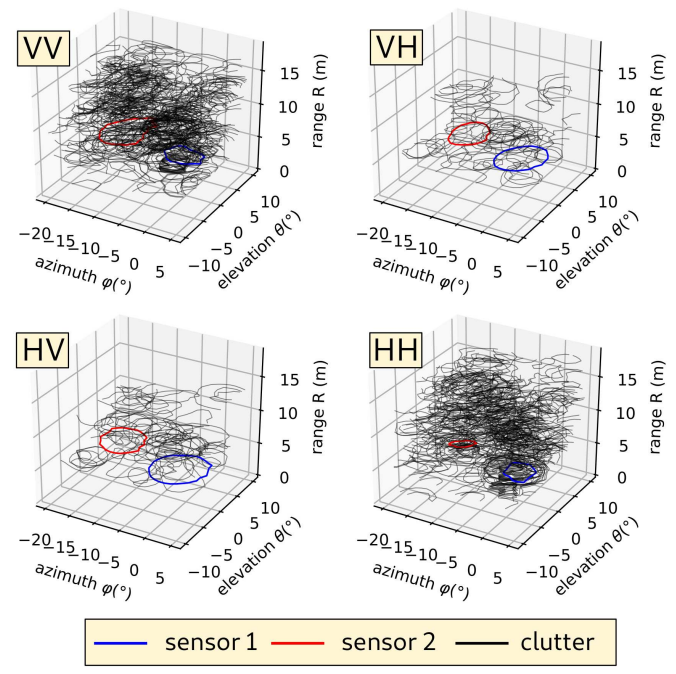

Fig. 4. Isolines (in elevation/azimuth/range coordinates) derived from the radar beamscanning of the scene and computed from different polarization configurations $(p=V V, V H, H V$, and $H H)$. In black color are displayed isolines of the clutter, while blue and red colors indicate isolines of the sensing modes for respectively the pressure sensor 1 (for an applied overpressure of 0,7 bar) and the pressure sensor 2 (for an applied overpressure of 1,1 bars).

$e_{\max , p}$ as the highest echo level inside the region enclosed by the isoline for the polarization configuration $p$. The variation of $e_{\max , p}$ as a function of the overpressure applied on the two sensors is displayed in Fig. 5. In Table 2, the full-scale measurement range $\Delta_{p}$ (with $p=V V, V H, H V$, and $H H$ ) is reported for the two sensors between 0.1 bar and 1.1 bars and are compared with the full-scale measurement range $\Delta_{S_{q}}$ (with $q=11,12,21$ and 22 ) of the scattering parameters derived from Fig. 3. As expected, the highest full-scale ranges are obtained from sensor 2, because its scattering parameters are more sensitive to overpressure variation, as it can be observed from Fig. 3. For each sensor, estimator $e_{\max , V V}$ differs from $e_{\max , H H}$ because $S_{11}$ differs from $S_{22}$. Moreover, the variations versus overpressure of $e_{\max , V H}$ and $e_{\max , H V}$ are similar because $S_{12}$ and $S_{21}$ are also similar for the two sensors (see Fig. 3). We observe an offset of $3 \mathrm{~dB}$ between $e_{\max , V H}$ and $e_{\max , H V}$ due to the unexpected difference of link budget between $R_{x, 1}$ and $R_{x, 2}$ channels. Last but not least $e_{\max , H H}$ is lower than the noise level when the applied overpressure is below 0,7 bar. To highlight the benefit of the $\mathrm{X}$-pol configuration compared with co-pol configuration, the sensitivity (or slope) of $e_{\max , p} \frac{\Delta_{p}}{\Delta_{S_{q}}}$ (units are in $\mathrm{dB} / \mathrm{dB}$ ) is also calculated between 0.1 bar and 1.1 bars and reported in Table 2. For the sensor 1 which has lower full-scale ranges, sensitivities are approximately the same for all polarization configurations, except for $p=H H$ which offers low sensitivity (0.2). We observe for the sensor 2, which has higher full-scale measurement ranges, that the sensitivity in $\mathrm{x}$-pol configuration is higher than one obtained from co-pol configurations ( 0.6 for $p=V V, 0.8$ for $p=V H$ and 1.1 for $p=H V)$.

To evaluate the measurement uncertainty of the overpressure, the beamscanning of the scene is performed successively 50 times. The mean value, standard deviation of 
Table 2. Full-scale range $\Delta_{S_{q}}$ of the scattering parameter $S_{q}$ (with $q=11$, 12,21 and 22), full-scale measurement range $\Delta_{p}$ (with $p=V V, V H, H V$, and $H H$ ) and sensitivity $\frac{\Delta_{p}}{\Delta_{S_{q}}}$ of the estimator $e_{\max , p}$ between 0.1 bar and 1.1 bars for the two passive pressure sensors.

\begin{tabular}{|c|c|c|c|c|c|c|}
\hline \multirow{2}{*}{$\mathrm{p} / S_{q}$} & \multicolumn{3}{|c|}{ pressure sensor 1 } & \multicolumn{3}{c|}{ pressure sensor 2 } \\
\cline { 2 - 7 } & $\Delta_{p}(\mathrm{~dB})$ & $\Delta_{S_{q}}(\mathrm{~dB})$ & $\frac{\Delta_{p}}{\Delta_{S_{q}}}$ & $\Delta_{p}(\mathrm{~dB})$ & $\Delta_{S_{q}}(\mathrm{~dB})$ & $\frac{\Delta_{p}}{\Delta_{S_{q}}}$ \\
\hline$V V / S_{11}$ & 2.1 & 3.1 & 0.6 & 3.1 & 4.5 & 0.6 \\
\hline$V H / S_{12}$ & 1.5 & 2.8 & 0.5 & 2.5 & 3.1 & 0.8 \\
\hline$H V / S_{21}$ & 1.6 & 2.8 & 0.5 & 3.6 & 3.1 & 1.1 \\
\hline$H H / S_{22}$ & 2.7 & 9.5 & 0.2 & $\mathrm{NR}^{a}$ & 7.6 & $\mathrm{NR}^{a}$ \\
\hline
\end{tabular}

${ }^{a} \mathrm{NR}$ : not relevant
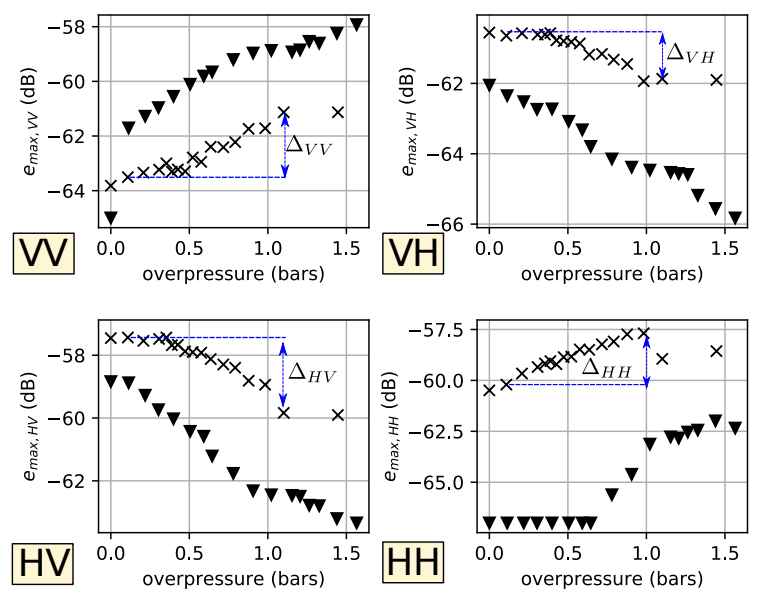

$$
\begin{array}{|ll|}
\times \text { sensor } 1 & \nabla \text { sensor } 2 \\
\hline
\end{array}
$$

Fig. 5. Statistical estimator $e_{\max , p}$ as a function of the applied overpressure for the pressure sensor 1 (crosses) and pressure sensor 2 (down triangles) and for the four polarization configurations $V V, V H, H V$, and $H H$.

the echo level and isolines are derived in linear scale from the volume used for Fig. 4. The mean value and standard deviation of $e_{\max , p}$, denoted $\operatorname{mean}\left(e_{\max , p}\right)$ and $\operatorname{std}\left(e_{\max , p}\right)$ are computed for each isoline and displayed in Fig. 6a in $\mathrm{dB}$ scale. It can be observed that mean $\left(e_{\max , p}\right)$ and $\operatorname{std}\left(e_{\max , p}\right)$ are strongly correlated, and mean $\left(e_{\max , p}\right)$ increases with $\operatorname{std}\left(e_{\max , p}\right)$. Let be $\epsilon_{p}$ the measurement uncertainty on the applied overpressure defined as follows:

$$
\epsilon_{p}=\frac{\operatorname{std}\left(e_{\max , p}\right)}{\operatorname{mean}\left(e_{\max , p}\right)}
$$

The uncertainty $\epsilon_{p}$ (in $\%$ ) is displayed in Fig. $6 \mathrm{~b}$ as a function of $\operatorname{mean}\left(e_{\max , p}\right) . \epsilon_{p}$ decreases with mean $\left(e_{\max , p}\right)$. As expected, the overpressure estimation derived from isolines of low radar echo level is less accurate than estimations obtained from isolines of higher echo level. However, compared with co-pol configurations, the impact on measurement uncertainty is strongly reduced for $\mathrm{x}$-pol configurations. As an example, when the overpressure of 0.5 bar is applied on the sensor 2, it can be observed from

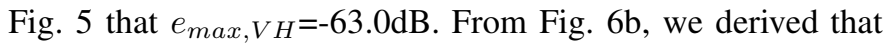
$2.5 \%<\epsilon_{V V}<7.5 \%$ and $2.5 \%<\epsilon_{V H}<5.0 \%$ at $-63 \mathrm{~dB}$. The measurement uncertainty decreases with $e_{\max , p}$ for both coand $\mathrm{x}$-pol configurations, but to a lesser extent for the $\mathrm{x}$-pol configuration due to higher signal-to-noise ratios.

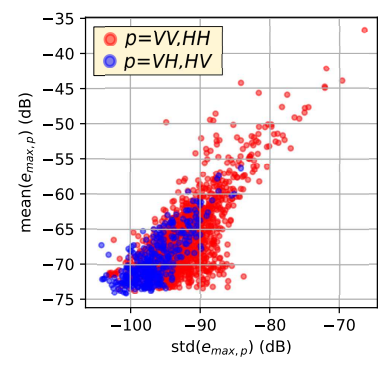

(a)

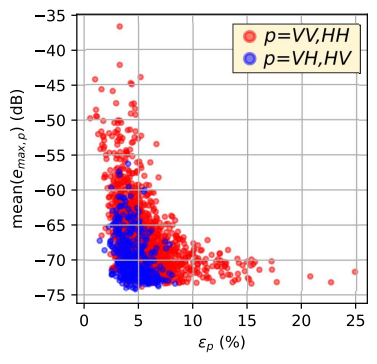

(b)
Fig. 6. $\operatorname{mean}\left(e_{\max , p}\right)$ as a function of (a) $\operatorname{std}\left(e_{\max , p}\right)$ and (b) of the measurement uncertainty $\epsilon_{p}$ defined by Eq. (2). Isolines are computed from fifty 3D radar beamscannings. Red and blue circles indicate isolines obtained from co-pol and x-pol configurations, respectively.

\section{CONCLUSION}

We have demonstrated in this paper that cross-polarized electric fields combined with a 3D radar imagery technique can be advantageously used to perform the long-range ( $>$ 3 meters) simultaneous interrogation of passive and wireless sensors in cluttered environments. Moreover, the 3D analysis of the clutter with isolines can be used to localize specific spots of a scene with high signal-to-noise ratio, and consequently may be advantageously used for avoiding shadow regions in the placement of the passive sensors.

\section{ACKNOWLEDGMENT}

The authors wish to thank Occitanie Region (CARANUC Project) and EDF (Electricité de France) for financial support. This work was partly supported by LAAS-CNRS micro and nanotechnologies platform members of the French RENATECH network.

\section{REFERENCES}

[1] M. Pereira da Cunha, R. J. Lad, T. B. Pollard, D. McCann, E. McCarthy, P. Prata, and R. Kelley, "Wireless harsh environment SAW array system for power plant application," in 2014 IEEE International Ultrasonics Symposium, Sep. 2014, pp. 381-384.

[2] H. Cheng, X. Ren, S. Ebadi, Y. Chen, L. An, and X. Gong, "Wireless passive temperature sensors using integrated cylindrical resonator/antenna for harsh-environment applications," IEEE Sensors Journal, vol. 15, no. 3, pp. 1453-1462, March 2015.

[3] A. J. Mercer, R. K. James, G. Bennett, P. Patel, C. Johnston, and J. Cai, "RFID testing and evaluation for an RF-harsh environment," in 2011 IEEE International Conference on RFID-Technologies and Applications, Sep. 2011, pp. 95-102.

[4] Y. Feng, L. Xie, Q. Chen, and L. Zheng, "Low-cost printed chipless RFID humidity sensor tag for intelligent packaging," IEEE Sensors Journal, vol. 15, no. 6, pp. 3201-3208, June 2015.

[5] A. Vena, E. Perret, and S. Tedjini, "A compact chipless RFID tag using polarization diversity for encoding and sensing," in 2012 IEEE International Conference on RFID (RFID), April 2012, pp. 191-197.

[6] D. Girbau, A. Ramos, A. Lazaro, S. Rima, and R. Villarino, "Passive wireless temperature sensor based on time-coded UWB chipless RFID tags," IEEE Transactions on Microwave Theory and Techniques, vol. 60, no. 11, pp. 3623-3632, Nov 2012.

[7] D. Henry, J. G. D. Hester, H. Aubert, P. Pons, and M. M. Tentzeris, "Long-range wireless interrogation of passive humidity sensors using van-atta cross-polarization effect and different beam scanning techniques," IEEE Transactions on Microwave Theory and Techniques, vol. 65, no. 12, pp. 5345-5354, Dec 2017.

[8] J. Philippe, M. V. De Paolis, D. Henry, A. Rumeau, A. Coustou, P. Pons, and H. Aubert, "In-situ wireless pressure measurement using zero-power packaged microwave sensors," Sensors, vol. 19, no. 6, 2019. 